\title{
Bio-Artificial Synergies for Grasp Posture Control of Supernumerary Robotic Fingers
}

\author{
Faye Y. Wu \\ Harry H. Asada \\ d'Arbeloff Laboratory, Massachusetts Institute of Technology \\ Cambridge, MA 02139, USA, \{yfwu, asada\} @ mit.edu
}

\begin{abstract}
A new type of wrist-mounted robot, the Supernumerary Robotic (SR) Fingers, is proposed to work closely with the human hand and aid the human in performing a variety of prehensile tasks. For people with diminished functionality of their hands, these robotic fingers could provide the opportunity to live with more independence and work more productively. A natural and implicit coordination between the SR Fingers and the human fingers is required so the robot can be transformed to act as part of the human body. This paper presents a novel control algorithm, termed "Bio-Artificial Synergies", which enables the SR and human fingers to share the task load together and adapt to diverse task conditions. Through grasp experiments and data analysis, postural synergies were found for a seven-fingered hand comprised of two SR Fingers and five human fingers. The synergy-based control law was then extracted from the experimental data using Partial Least Squares (PLS) regression and tested on the SR Finger prototype as a proof of concept.
\end{abstract}

Keywords-wearable robots; supernumerary robotic limbs; grasping; postural synergy; Partial Least Squares regression

\section{INTRODUCTION}

Wearable robots represent a special class of robots that are physically attached to the human and perform tasks closely with the wearer. Two types of wearable robots have already been studied extensively: exoskeletons and prosthetic devices. The former extends the joint strength of the human body by attaching actuators to individual human joints and the latter substitutes lost human limbs with mechanical proxies. Recently, a third type of wearable robots, Supernumerary Robotic (SR) Limbs, has been explored. The SR Limbs are attached directly to the human body to aid the user in holding objects, supporting the body weight, sharing a workload, and streamlining the execution of a task. For example, WaistMounted robotic arms not only can hold extra tools for a worker when performing manufacturing and assembly tasks, but also can brace the human body against a wall or a surrounding structure to reduce the load on the knee and hip joints while consuming minimal energy [1-3]. Another example is Robot-on-the-Shoulder, which can assist the human in lifting and holding an object in the overhead area, allowing a worker to carry out overhead installation and maintenance jobs ${ }^{1}$ with ease [4-5].

*Experiments reported in this paper are performed following COUHES approved protocol \# 1402006178.
This paper discusses another type of SR Limbs: Supernumerary Robotic Fingers. SR Fingers are attached to the wrist of a human to extend the functionality and capability of the five human fingers. These robotic fingers can assist the human in performing difficult tasks, such as grasping a large or oddly-shaped object, and holding an object while the human fingers perform a dexterous task on the object, e.g. holding a tablet computer while typing letters on it or holding a water bottle while taking the cap off.

Integrating the motion of the SR Limbs with that of the human limbs is a critical challenge. Compared to conventional control between a separate, self-standing robot and a human, more natural and implicitly coordinated control is required for the SR Limbs so the human can come to perceive the robotic limbs as an extension of his/her own body, although they are biologically uncoupled. This implies that the SR limbs can effectively assist the human without requiring explicit commands. Rather, the motion of SR limbs is linked to the human limbs, conforming to a functional relationship with the human limbs in a way that is perceived to be natural to the human. Here, we present a novel control algorithm, termed "Bio-Artificial Synergies", inspired by neuromotor control to coordinate the combination of the innervated human limbs and non-innervated SR Limbs such that they share the task load and adapt to diverse task conditions.

Since the concept of synergies was proposed in 1967 [6], it has been studied extensively in neurophysiology and experimental brain science. In the context of locomotion and motor control, synergies are defined as groups of muscles jointly activated by a single central control signal [7]. This coherent activation, in space or time, of the muscle groups is much more efficient than directly controlling all of the redundant muscles of the body [8]. Through the sequencing and superposition of only a small number of these muscle synergies, a large variation of complex, multiphasic movements can be accomplished [9, 10]. Muscle synergies have been used to explain limb trajectories and gait modifications in frogs [11, 12] and cats [13]. Synergies have also been identified in human motion during reaching movements [14-16], turning and balancing [17], as well as forward and backward walking $[18,19]$. Prehensile tasks alone have led to a wealth of literature on postural and forcestabilizing synergies [20-25].

Synergies have also been utilized in the realm of robotics control and design. They were used for controlling upper arm 
neuroprosthesis [26] to significantly simplify the multi-DOF motion. Grasp synergies [20] were applied to robotic hand design [27-30] and grasp planning and control [31-38]. Alternatively, nonlinear oscillators have used synergies to learn and reproduce complex rhythmic movements [39, 40].

To achieve the above mentioned "natural and implicit coordination", we first explored the concept of postural synergy between the human limbs and the SR Limbs, in particular concentrating on grasping postures. We describe this type of synergy as "Bio-Artificial" to differentiate it from those reported in neurophysiology literature, since nerves do not extend to the SR Limbs. This paper briefly presents the design concept of the SR Fingers and mainly focuses on the synergybased control that enables the desired coordination between the robot and human fingers. In the preliminary design, the two SR Fingers are attached to the human wrist to form a 7-fingered hand. The postural synergies among the seven fingers are analyzed using Principal Components Analysis (PCA) and Partial Least Square (PLS) regression is used to correlate the human finger movements with the robotic finger movements in order to create a tightly coordinated control. The experimental data of actual grasping tests were analyzed to validate the datadriven methodology for coordinating human-robot fingers.

\section{SUPERNUMERARY ROBOTIC FINGERS: A DESIGN CONCEPT}

When individuals have diminished dexterity and range of motion in their hands due to aging or disabilities, it can be difficult for them to perform routine tasks of living, such as taking the lid off a jar or picking up a pot of plant. According to the US Census, there are at least 52.6 million people currently suffering from some extent of dexterity and limb mobility impairment [41]. Although a range of assistive technologies are available, they provide only limited or specific assistances, e.g. a specialized grip on a pen for easy grasping and automatic card shufflers. High-tech assistive devices, such as specially adapted computers, are more useful, but few are ready to use directly out-of-the-box. Considerable end-user training is required for those devices.

It would be ideal to have a device that operates seamlessly with the human hand and effortlessly fulfills a multitude of functions, as if it were part of the human body. SR Fingers that are mounted to the human hand could complement the existing human fingers and enable the user to more comfortably and easily perform manual tasks. Since the SR Fingers are not as limited in size, range, and motion as the human fingers are, they are especially beneficial for grasping objects that are usually deemed difficult to grip, including objects that are large, oddly-shaped, heavy, slippery, too hot, or too cold. The SR Fingers can also help the user to perform tasks that usually require two hands, such as zipping up a zipper or plugging in a battery charger, with a single hand. This gives the SR Fingers the potential to serve as prosthetic devices, replacing or supplementing traditional prostheses. Additionally, tools can be attached to the tips of the SR Fingers to facilitate tasks that require more than the bare hands. Fig. 1 illustrates some examples of possible configurations and applications of the SR Fingers.
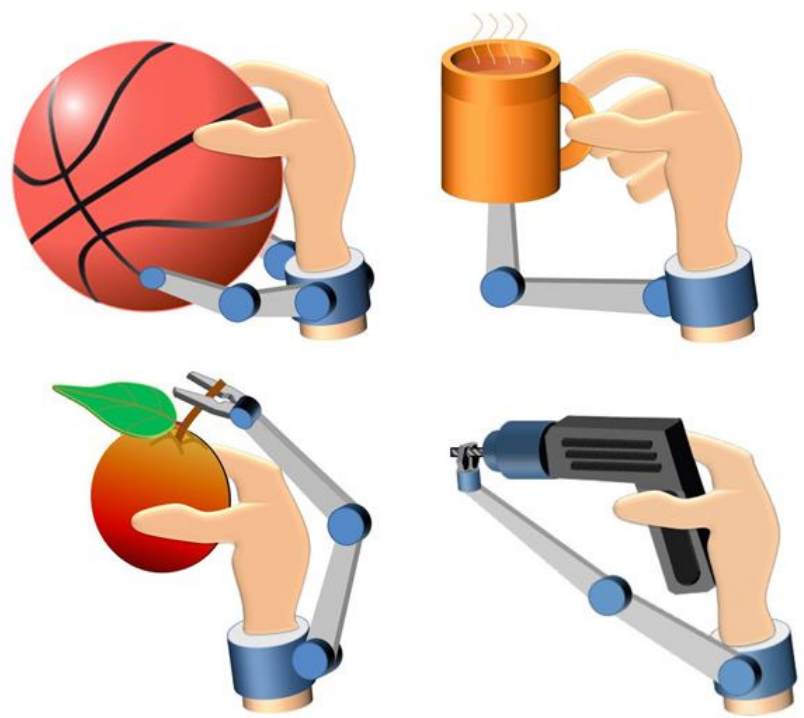

Figure 1. SR Fingers could provide the assistance needed to more comfortably and securely grasp objects and perform tasks.

In anticipation of circumstances where only the SR Fingers are used to carry the weight of an object while the human fingers perform more precise and delicate actions, we designed a pair of SR Fingers that mimics the human grasping action [42]. To provide the robotic fingers with the largest workspace possible around the human hand while keeping the design relatively simple, the two SR Fingers are fixed to opposite sides of the wrist, as depicted in Fig. 2. Each SR Finger has 3 DOF, with RT moving similarly to the human thumb (including finger circumduction, abduction, and flexion) and RF similar to one of the long fingers (including abduction and two flexion movements). The SR Fingers have fewer DOFs than the actual fingers being mimicked, which enable easier prototyping and control while still allow for various grasping postures.

The size of the SR Fingers and their range of motion are

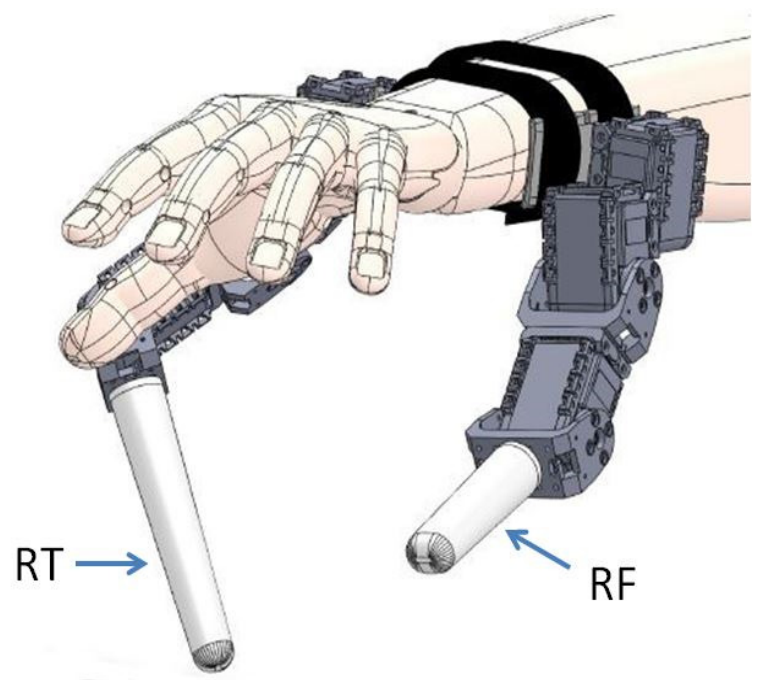

Figure 2. SR Fingers are mounted on the human wrist to form a 7-fingered hand: 5 human fingers and 2 robotic fingers. 
greater than those of the human fingers to account for large objects and complex tasks. The human index finger is used as a base line, which has the greatest range of motion $\left(20^{\circ}\right.$ abduction and $80^{\circ} \sim 110^{\circ}$ flexion) [43] and a reported maximum fingertip force of 5.7N [44]. The SR Fingers, after testing a few different sizes and configurations next to common objects, were selected to have $300^{\circ}$ of movement in the proximal joint and $200^{\circ}$ movement in the other two joints, where RT is $200 \mathrm{~mm}$ long and RF $220 \mathrm{~mm}$ long, to cover a large operation space. The first two moving links of the SR Fingers consist of only the actuators (32 mm x $50 \mathrm{~mm} \times 40 \mathrm{~mm})$, as shown in Fig. 2, to keep the center of mass close to the wrist and limit the actuators' inertial contribution to the finger movement. These actuators, Dynamixel AX-12A servos (Robotis, South Korea), are rated at a maximum torque of $1.5 \mathrm{Nm}$ to enable the SR Fingers to sustain fingertip forces similar to those experienced by the human fingers during grasping motion [44]. The last link is $70 \mathrm{~m}$ and $90 \mathrm{~m}$ long for RT and RF, respectively, where the breadth and depth ratio of the two ends of the link follows that of the human finger [45]. This preliminary design serves as a proof of concept for wearable robotic fingers and a test bed for human-robot coordination control. Feasibility studies of different finger layout and optimization of the mechanical design are to be investigated beyond the proof of concept stage and are thus out of the scope of this paper.

\section{BIO-ARTIFICIAL SYNERGIES}

As mentioned earlier, postural synergies were found in 5fingered hands during prehensile actions [20]. The question is whether we can apply the same principle to a 7-fingered hand to achieve postures that feels and looks natural to the user. To address this issue, we captured static grasping data of a 7fingered hand using a protocol similar to the one presented in [20] and synthesized the most significant variables that explain the data variance with PCA.

The modeled 7-fingered hand in Fig. 2 was used to simulate grasping in SolidWorks (SolidWorks, Waltham, MA) of 8 objects with diverse shapes and sizes. The objects were selected so all 7 fingers can be used to perform the grasp, and as a result small objects such as pencils or cards were not used. The modeled fingers were moved individually until the user subjectively decides that the best grasping position has been reached. Five trials were performed for each object, where the posture and grasp locations varied between trials, as shown in Fig. 3.

Similar to [20], the 7-fingered hand grasping posture was tested without force feedback so the general form of the posture is only affected by the geometry of the hand and not by external factors, such as contact forces and slippage. The purpose of the posture evaluation is to extract human preferences in positioning fingers, which is subconsciously known to the users but difficult to describe. Solidworks is not ideal for generating stable grasps, but it is a platform that allows manual manipulation of fingers and viewing of the posture from different angles, which helps the users determine what looks "natural" to them. Further analysis involving force interaction and stability will enhance grasp performance of the SR Fingers, but it is not included in this paper as only the
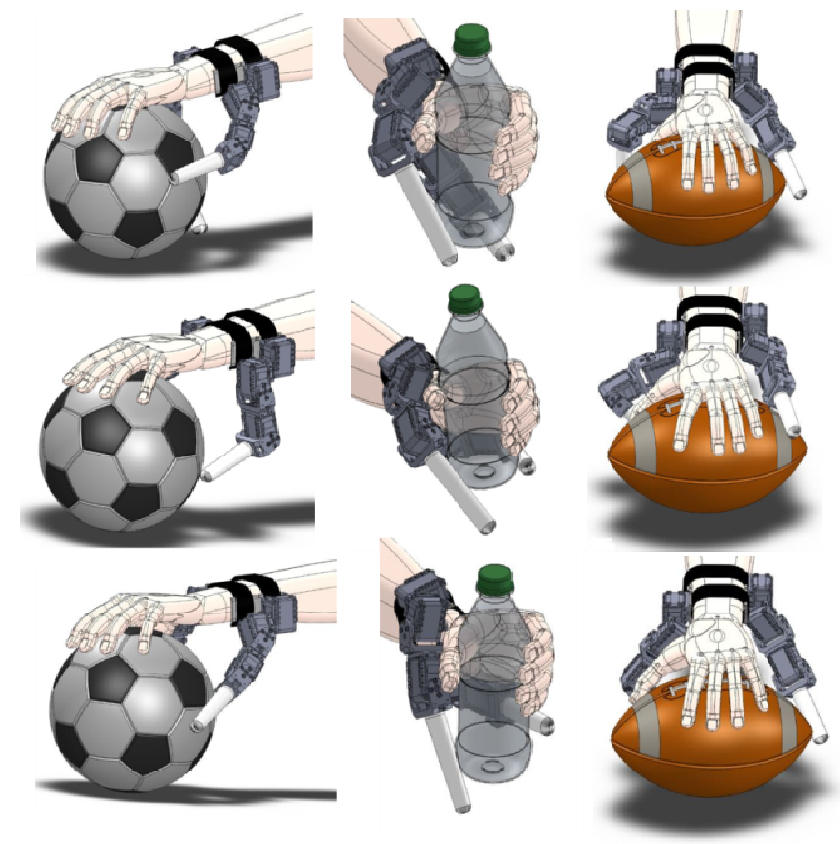

Figure 3. Various objects were grasped at diverse locations to gather data for synthesizing postural synergies of a 7-fingered hand.

general form of grasping postures are being investigated at this stage.

We measured 19 joint angles for the human fingers and 6 for the robotic fingers. The inward circumduction of the thumb, the abduction of each finger, as well as the flexion of the metacarpophalangeal and interphalangeal joints were defined as positive; the joint angles were set to $0^{\circ}$ when the fingers were straight, close together, and in plane with the palm. Angles of the robotic finger joints were defined as $0^{\circ}$ when RF was pointing straight forward like the human fingers, and RT was in plane with the palm but perpendicular to the wrist.

PCA was selected for analyzing the grasping data because it is particularly effective at dealing with situations with large number of system variables $(r=19+6=25)$ and small number of observations $(N=40)$. The data was put into a matrix $\boldsymbol{X}$

$$
\mathbf{X}=\left[\mathbf{x}^{1}, \cdots, \mathbf{x}^{N}\right] \in \mathfrak{R}^{r \times N},
$$

where $\mathbf{x}^{i}=\left[x_{1}, \cdots, x_{r}\right]^{T}$ is a sample of mean-centered joint angles. Using the eigenvectors $\left(\boldsymbol{v}_{\boldsymbol{i}}\right)$ and eigenvalues $\left(\lambda_{\mathrm{i}}\right)$ of the covariance of $\boldsymbol{X}$, we can approximate $\boldsymbol{X} \boldsymbol{X}^{\mathrm{T}}$ with

$$
\mathbf{X} \mathbf{X}^{T} \cong \lambda_{1} \mathbf{v}_{1} \mathbf{v}_{1}{ }^{T}+\lambda_{2} \mathbf{v}_{2} \mathbf{v}_{2}{ }^{T}+\cdots+\lambda_{s} \mathbf{v}_{s} \mathbf{v}_{s}{ }^{T},
$$

where $s \leq r<N$. The accuracy of this approximation represents the data percentage variance accounted for by the PCs. It is evaluated with the eigenvalues as

$$
\mu=\frac{\lambda_{1}+\lambda_{2}+\cdots \lambda_{s}}{\lambda_{1}+\lambda_{2}+\cdots \lambda_{r}} \times 100 \% .
$$

Fig. 4 illustrates the calculated $\mu$ for each of the first 6 PCs. For the 7-fingered hand, the first two PCs together can account for approximately $82 \%$ of the variance, which suggests that 
grasping postures of a 7-fingered hand can be produced with much fewer DOFs, reducing from 25 to 2 or 3 . This is consistent with the human grasp synergy. Since the number of data sample is at least 10 times larger than the number of meaningful variables, the dominant PCs for grasping postures that require all 7 fingers are believed to be sufficiently approximated in this analysis. Fig. 4 also includes the result of PCA performed on the joint angles of the human fingers lone. Although the percentage variance shown is slightly higher than the average value reported in [20], it is comparable to most values found in actual subjects' tests. In both 5-fingered and 7fingered data analysis, PCA revealed that the first component of the grasping postural synergy represents in-phase motion of the thumb and the other four fingers, while the second component indicates out-of-phase motion. Interestingly, the 2 robotic fingers, too, exhibited similar patterns of motion: the first component is in-phase motion of the two SR Fingers, while the second component is out-of-phase motion. SolidWorks models were also constructed with the SR Fingers mounted further down the forearm, and PCA results were similar to those shown in Fig. 4. Although the coefficients of the specific PCs changed, the general in-phase and out-ofphase motion represented by the first two PCs remained the same.

Since the two SR Fingers were designed to take the role of the thumb and an ordinary finger, it was hypothesized that RT would be more correlated with the human thumb than with the other four fingers and vice versa for RF. This was verified by performing PCA on two separate data groups, one consists of the thumb and RT measurements, and the other consists of data from the rest of the human fingers and RF. As shown in Table 1 , the percentage variances explained by the PCs of each subgroup are higher than those explained by the PCs of the entire data set. Especially for the thumb and RT combination, the variances accounted for by the first two PCs increased $10 \%$. Although the subgroup containing RF and the other four human fingers only exhibited slight improvement, it still means that the thumb and RT had little effects on the rest of the fingers. For comparison purposes, the data of the thumb and the other four fingers were also separated for the 5-fingered hand and the PCA result showed similar improvement. These findings

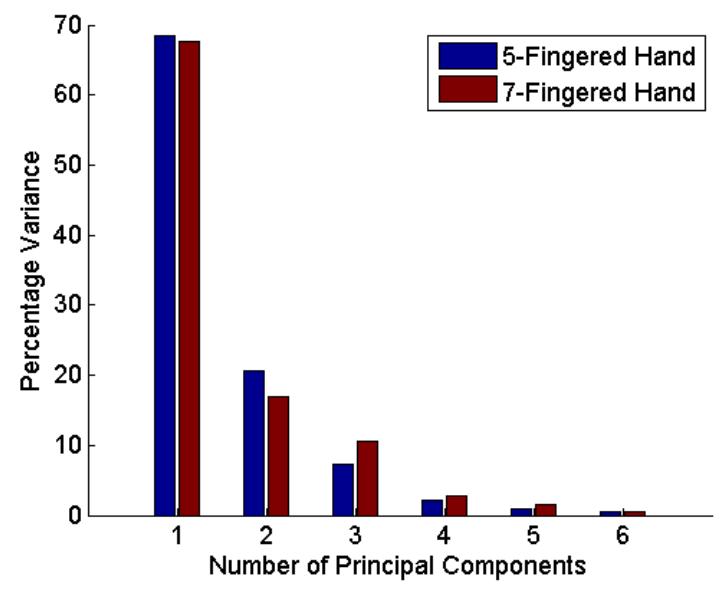

Figure 4. Data variance explained by each principal component for 5fingered hand and 7-fingered hand.
TABLE I. DATA VARIANCE EXPLAINED By SUB-SYNERGY GROUPS

\begin{tabular}{|c|c|c|c|c|}
\hline \multirow{3}{*}{$\begin{array}{c}\text { Principal } \\
\text { Components }\end{array}$} & \multicolumn{4}{|c|}{ Percentage Variance } \\
\hline & \multicolumn{2}{|c|}{ 5-Fingered Hand } & \multicolumn{2}{|c|}{ 7-Fingered Hand } \\
\hline & Thumb & 4 Fingers & $T h u m b+R T$ & 4 Fingers $+R F$ \\
\hline \multirow{2}{*}{ PC1 } & 77.8 & 69.7 & 73.1 & 70.3 \\
\hline & \multicolumn{2}{|c|}{66.6} & \multicolumn{2}{|c|}{65.5} \\
\hline \multirow{2}{*}{$\mathrm{PC} 2$} & 20.3 & 18.5 & 21.2 & 15.6 \\
\hline & \multicolumn{2}{|c|}{20.6} & \multicolumn{2}{|c|}{16.9} \\
\hline \multirow{2}{*}{$\mathrm{PC} 1+\mathrm{PC} 2$} & 98.1 & 88.2 & 94.3 & 85.9 \\
\hline & \multicolumn{2}{|c|}{87.2} & \multicolumn{2}{|c|}{82.4} \\
\hline
\end{tabular}

suggest that sub-synergies exist within the four fingers, and the thumb forms another set of sub-synergies separately. These sub-synergies are also extended to the SR Fingers, which may help with correlating and coordinating the movements between the human hand and the robotic fingers.

\section{COORDINATION CONTROL BASED ON PARTIAL LEAST SQUARES REGRESSION}

The Bio-Artificial postural synergy of the 7-fingered hand analyzed with PCA suggests that as long as the task is to enclose a class of objects with all 7 fingers, the motion of the SR Fingers is strongly correlated with the motion of the human fingers. Our objective, however, is not merely to find postural synergy of augmented fingers, but also to obtain a useful control law for natural and implicit coordination between mechanical and biological limbs. Therefore, we need to be able to predict the posture of the SR Fingers based on the posture of the human fingers. To that end, we re-formulated the PC-based analysis by splitting the experimental data into an input matrix $\boldsymbol{X}$, consisting of data from only the 5 human fingers, and an output matrix $\boldsymbol{Y}$, consisting of data from the 2 SR Fingers.

$$
\mathbf{X}=\left[\mathbf{x}^{1}, \cdots, \mathbf{x}^{N}\right] \in \mathfrak{R}^{n \times N}, \mathbf{Y}=\left[\mathbf{y}^{1}, \cdots, \mathbf{y}^{N}\right] \in \mathfrak{R}^{m \times N},
$$

where $\mathbf{x}^{i}=\left[x_{1}, \cdots, x_{n}\right]^{T}$ is a sample of mean-centered joint angles of the human fingers and $\mathbf{y}^{i}=\left[y_{1}, \cdots, y_{m}\right]^{T}$ is that of the SR Fingers $(n=19, m=6)$.

Similar to PCA, we consider a unit vector in the input space $\mathbf{v} \in \Re^{n \times 1}$ and the projection of each input sample onto the unit vector is $z^{i}=\mathbf{v}^{T} \mathbf{x}^{i}, i=1, \cdots, N$. Unlike PCA, we also consider a unit vector in the output space $\mathbf{w} \in \mathfrak{R}^{m \times 1}$ and the projection of each output sample onto the vector is $s^{i}=\mathbf{w}^{T} \mathbf{y}^{i}$. These projections are called latent variables. We can find the specific directions of the two unit vectors that maximize the correlation between the two latent variables with

$$
\mathbf{v}^{o}, \mathbf{w}^{o}=\arg \max _{\substack{|\mathbf{v}|=1 \\|\mathbf{w}|=1}} \sum_{i=1}^{N} z^{i} s^{i}=\arg \max _{\substack{|\mathbf{v}|=1 \\|\mathbf{w}|=1}} \mathbf{v}^{T} X Y^{T} \mathbf{w},
$$

The solution is given by the following: $\mathbf{v}^{o}$ is the unit eigenvector associated with the maximum eigenvalue of matrix $X Y^{T} Y X^{T}$, while $\mathbf{w}^{o}$ is the unit eigenvector associated with the maximum eigenvalue of matrix $Y X^{T} X Y^{T}$. This pair of 
eigenvectors gives the most significant component in correlating the input and output data. This is the key concept of Partial Least Squares (PLS) regression [46].

The complete algorithm provides a series of unit vectors in the order of descending significance. Repeating the same process for the residue of the data until the output can be well predicted by input components, we can obtain a control law that maximally correlates the posture of two robotic fingers with the posture of the five human fingers. It is in the form of:

$$
\mathbf{y}=\mathbf{x A},
$$

where matrix A contains information of all the eigenvectors of both input and output data matrices as well as the correlation strength of each pair of input and output latent variables.

To visualize the result of PLS analysis of the 7-fingered hand, Fig. 5 illustrates the first and second PLS components in both input and output spaces. Similar to the PCs, the first 2 PLS components represent in-phase and out-of-phase motion of the fingers. The difference between PCA and PLS is that PCA treats the correlations between all variables equally while PLS emphasizes on the correlations between input variables and output variables. Taking into account of circumstances where a component is small in the input space but it is highly correlated with some output components, PLS can make accurate predictions based on input-out correlation. As a result, however, the first few PLS components tend to account for less variance in the entire data set than the PCs: the first two

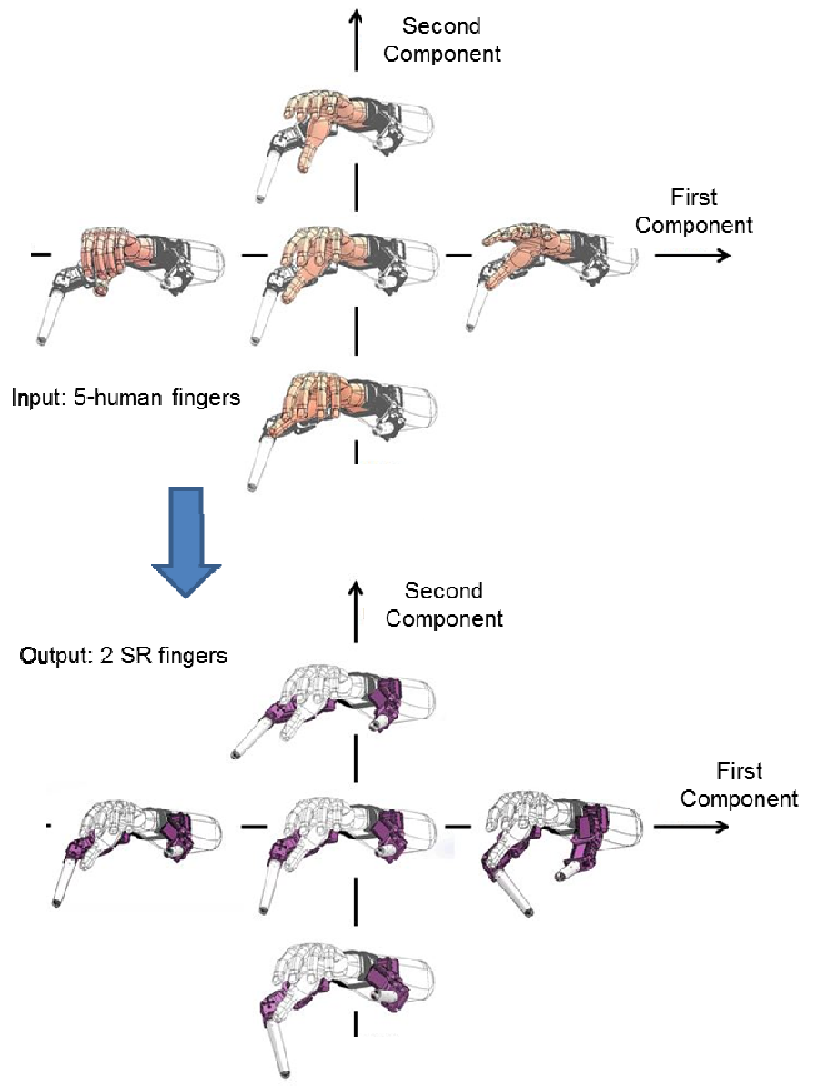

Figure 5. First two components of PLS analysis: The posture of the 2 SR fingers is correlated to the measured posture of the 5 human fingers. components of PLS account for approximately $80 \%$ of the total data variance (Table 2), whereas the first two PCs explain $84 \%$ of variance. Splitting the data into subgroups helps increasing the amount of data variance the components account for, although the improvement is not as significant as those observed in PCA. Based on the tabulated results, the synergybased postural control can be reasonably accomplished by PLS. Furthermore, if sub-synergies are used to control the movement of SR Fingers, i.e. using the thumb motion to predict the motion of RT and the other fingers for RF, only 2 or 3 PLS components would be sufficient to coordinate robotic fingers in concert with the human fingers.

TABLE II. DATA VARIANCE EXPLAINED IN INPUT AND OUTPUT SPACES FOR THE WHOLE DATA SET AND SUB-SYNGERY GROUPS

\begin{tabular}{|c|c|c|c|c|c|c|}
\hline \multirow{2}{*}{ Synergy Group } & \multicolumn{2}{|c|}{ PC1 } & \multicolumn{2}{|c|}{ PC2 } & \multicolumn{2}{|c|}{ PC1+PC2 } \\
\hline & Input & Output & Input & Output & Input & Output \\
\hline \multirow{2}{*}{$\mathrm{X} \rightarrow \mathrm{Y}$} & 68.2 & 64.6 & 20.8 & 6.1 & 89.0 & 70.7 \\
\hline & \multicolumn{2}{|c|}{66.4} & \multicolumn{2}{|c|}{13.4} & \multicolumn{2}{|c|}{79.8} \\
\hline \multirow{2}{*}{ Thumb $\rightarrow$ RT } & 77.8 & 70.7 & 20.2 & 2.8 & 98.0 & 73.5 \\
\hline & \multicolumn{2}{|c|}{74.2} & \multicolumn{2}{|c|}{11.5} & \multicolumn{2}{|c|}{85.8} \\
\hline \multirow{2}{*}{ Finger $\rightarrow$ RF } & 69.7 & 67.8 & 14.2 & 10.0 & 83.9 & 77.8 \\
\hline & \multicolumn{2}{|c|}{68.7} & \multicolumn{2}{|c|}{12.1} & \multicolumn{2}{|c|}{80.8} \\
\hline
\end{tabular}

As mentioned earlier, since only general grasping postures are being analyzed here, the SR Finger positions predicted by PLS only forms the general grasping shape in correspondence to human finger positions. To guarantee that an optimal and stable 7-finger grasp is actually performed on an object, more details, for example contact force synergies or joint torque synergies, need to be included in the synergy-based control. Since the goal of this paper is to prove that the SR Fingers can move in concert with the human fingers in a natural and implicit manner, postural synergy alone is sufficient.

\section{PROTOTYPING AND EXPERIMENT}

To confirm the Bio-Artificial synergies found in the SolidWorks models and validate the performance of the synergy-based control scheme of a 7-fingered hand, we constructed a prototype of the SR Fingers, which is shown in Fig. 6a. The robotic fingers are mounted on the wrist of the user with a $3 \mathrm{D}$ printed (fused deposition modeling) brace. The weight of the device $(<250 \mathrm{~g})$ is evenly distributed along the wrist and part of the forearm for better comfort.

We executed actual "grasps" while wearing the prototype SR Fingers on 20 objects of common shapes and sizes, as shown in Fig. 6b, where the fingers are close to but not touching the surface of the object (again, to extract the postural data that are unaffected by forces). Five trials were performed for each object and the average of the trials was used for analysis. The human hand posture was measured by a ShapeHand data glove (Measurand, Toronto, Canada), which contains fiber optics sensors that can precisely capture the positions of the wrist, the finger tips, and finger joints. The robotic fingers were moved by the user via LabVIEW (National Instruments, Austin, TX) until the "best" grasping position is reached. This "best" position is subjectively decided by the user, and it changes between trials as the human fingers 


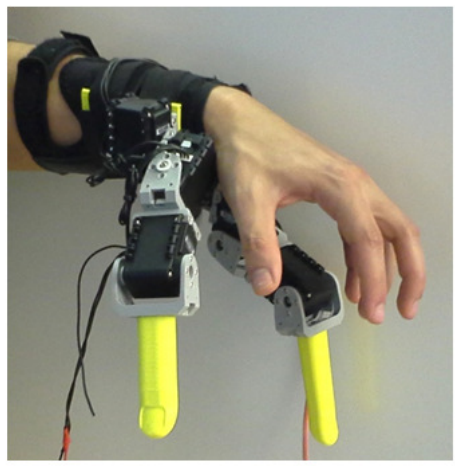

(a)

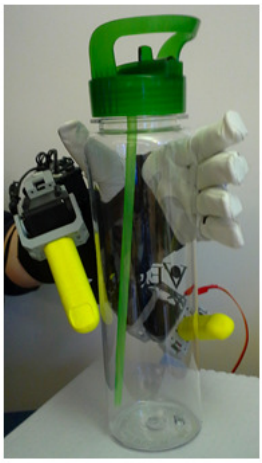

(b)
Figure 6. (a) Prototype of the two robotic fingers mounted on the human wrist. (b) Grasping experiment of the 7-fingered hand was conducted using a data glove.

approach the object at different locations.

The PCA result of the experimental data reveals that the first two PCs account for $76 \%$ of the variance, which is lower than the result presented in Fig. 4. This could be caused by the physical differences between the model and the subject's hand, as well as the distinct grasping habit of the subject that was not translated into the models. Nevertheless, there is a significant reduction of DOFs needed to form grasping postures, justifying the Bio-Artificial postural synergy for a 7-fingered hand. It is interesting to note that although the PCs of a 5-fingered hand also accounted for lower percentage variance than the models' $\mathrm{PCs}$, the value happens to be the same as the average value reported in [20].

Sub-synergy groups were also found in actual grasping tests. Just like before, the measurements for the thumb and RT were grouped separately from the rest of the measurements, and the percentage variance accounted for by the subgroups' PCs increased (Table 3). The improvement is much more obvious in the experimental data, with the first two PCs accounting for over $90 \%$ of data variance in both groups. Furthermore, the first two PCs of the subgroups for the 5fingered hand accounted for almost $100 \%$ of all data variance, which definitively verifies the existence of sub-synergies in the human hand.

Using PLS to predict the position of robotic fingers based on the human finger positions, we employed 3 PLS components, which accounted for $77 \%$ of variance in the

TABLE III. EXPERIMENTAL DATA VARIANCE EXPLAINED BY SubSYNERGY GROUPS

\begin{tabular}{|c|c|c|c|c|}
\hline \multirow{3}{*}{$\begin{array}{c}\text { Principal } \\
\text { Components }\end{array}$} & \multicolumn{4}{|c|}{ Percentage Variance } \\
\hline & \multicolumn{2}{|c|}{ 5-Fingered Hand } & \multicolumn{2}{|c|}{ 7-Fingered Hand } \\
\hline & Thumb & 4 Fingers & $T h u m b+R T$ & 4 Fingers $+R F$ \\
\hline \multirow{2}{*}{ PC1 } & 67.3 & 80.6 & 54.9 & 78.8 \\
\hline & \multicolumn{2}{|c|}{66.9} & \multicolumn{2}{|c|}{62.0} \\
\hline \multirow{2}{*}{$\mathrm{PC} 2$} & 32.2 & 17.5 & 35.5 & 12.0 \\
\hline & \multicolumn{2}{|c|}{17.6} & \multicolumn{2}{|c|}{14.3} \\
\hline \multirow{2}{*}{$\mathrm{PC} 1+\mathrm{PC} 2$} & 99.6 & 98.2 & 90.4 & 90.8 \\
\hline & \multicolumn{2}{|c|}{84.5} & \multicolumn{2}{|c|}{76.3} \\
\hline
\end{tabular}

output space $(88 \%$ variance for both input and output variables). Fig. 7 is an example of the error between the predicted SR Finger joint angles and the actual angles the user chose. In general, most of the error seems to be in joint 4 and 5, which are the first two joints of RF. Since the user can choose the RF to either support an object from the bottom or from the side, the orientation of RF varies significantly, even between grasping trials of the same object. The position of RT on the other hand is usually in the same direction as the human thumb, hence the errors are smaller. Fig. 8a shows the distribution of the prediction error for the entire data set, and almost $60 \%$ of the cases exhibited error less than $\pm 10^{\circ}$, which is acceptable for the purpose of defining general grasping postures. PLS was also performed on subgroups of variables, separately predicting the position of RT with the thumb measurements and RF with the other four fingers. The prediction error using sub-group synergies is much smaller across all 6 output variables, as shown in the example plotted in Fig. 7. This example is not a rare occurrence, since Fig. $8 \mathrm{~b}$ points out that over $80 \%$ of the cases had an error less than $\pm 10^{\circ}$.

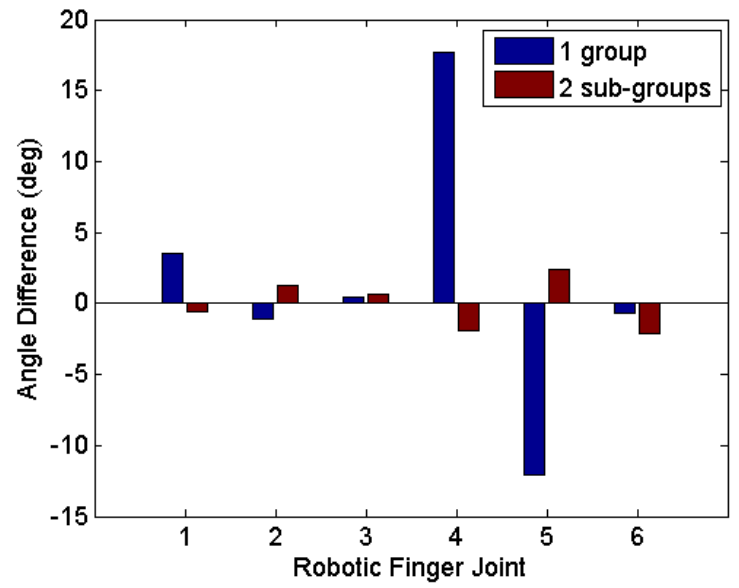

Figure 7. RS Finger joint angles are predicted based on human hand grasping posture for one object (PCV pipe) using 3 PLS components. The prediction error is smaller when the data is divided into sub-synergy groups.

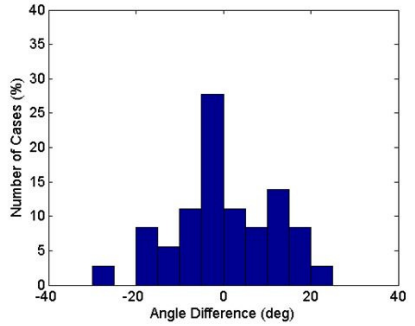

(a)

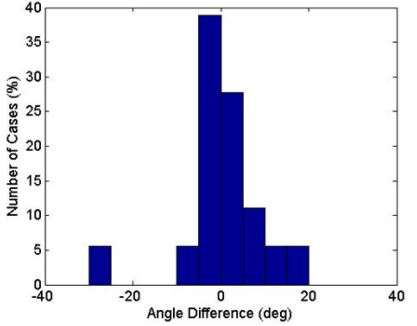

(b)
Figure 8. Prediction error distribution for RS Finger joint angles, when 3 PLS components are used, for all objects. (a) Error predicted using the entire data set. (b) Error predicted using sub-synergy groups.

Finally, to demonstrate the usefulness of the SR Fingers and the effectiveness of the synergy-based control, we implemented a simple data glove by attaching 3 stretch sensors (StretchSense, New Zealand) to the human hand, one on the thumb, one on the index finger, and one on the middle finger (simulating 3 PCs) and streaming live finger bending data into 


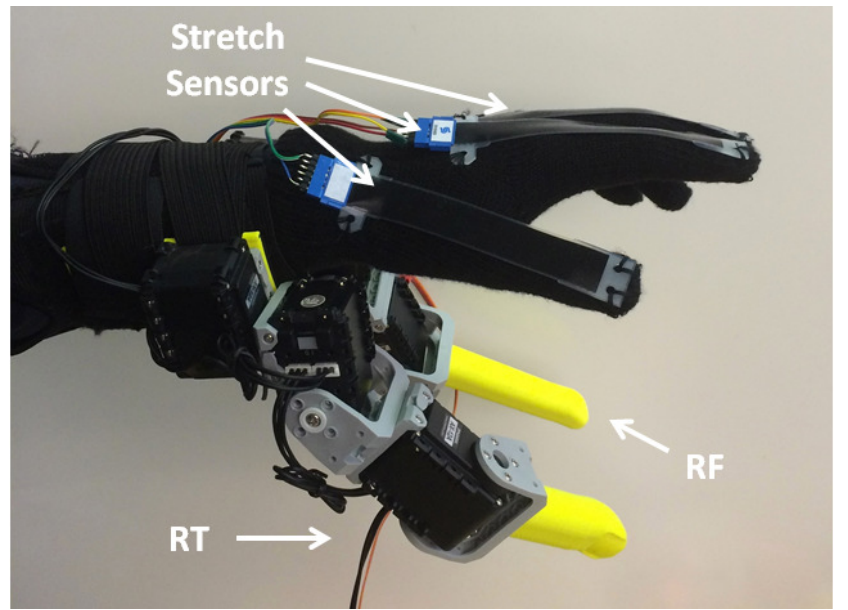

Figure 9. Using only three stretch sensors, the motion of the SR

Fingers can be coordinated with that of the human fingers.

LabVIEW to directly control the position of the SR Fingers (Fig. 9). PLS was performed to relate the stretch sensor reading to the ShapeHand measurements and the robotic finger joint angles. With only three sensors, the motion of the SR Fingers can already be coordinated with the motion of the human fingers to perform synchronized in-phase and out-of-phase movements, similar to those shown in Fig. 5. Using servo torque limits to help detect contact, we performed a few common grasp tasks with this setup. Shown in Fig. 10, a person with small hands can grab a large ball single handedly with the assistance of the SR Fingers, which is almost impossible for the user to do normally. The SR Fingers can also help the user to grasp objects in different ways (Fig. 11). And lastly, after the robotic fingers reach the desired positions, a hold function can be initiated, allowing the user to perform 2-handed tasks with a single hand, for example typing on a tablet computer (Fig. 12, the glove was removed to enable typing).

\section{CONCLUSION}

The synergy-based coordination control presented in this paper provides a novel and effective algorithm for correlating SR Fingers with the human fingers in a natural and implicit manner. The Bio-Artificial synergies generate low-level behaviors that play the key role in transforming a robot to act as part of the human body. They are essential in bringing innervated and non-innervated limbs together to share the work load, compensate for one another, and adapt to various tasks.

So far, we considered only the joint angles of each finger, but finger force and torque play an important role in grasp stability too $[47,48]$. Our next step is to determine correlation of fingertip forces between SR and human fingers. Control of contact forces will be more effective than merely controlling the hand posture in adapting to the irregularity and uncertainty of the object, hence enabling better grasp stability as reported in the literature of human fingers [21-25].

Bio-Artificial grasp synergies of the combined human-SR Fingers depend on the number of SR Fingers used, their size and shape, as well as the position and orientation of where the SR Fingers are attached to the human. It is important to find the right finger morphology and attachment configuration for

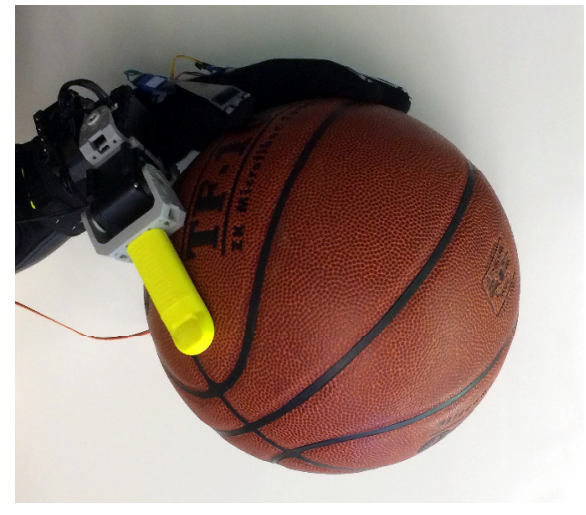

Figure 10. Normally a person with small hands cannot grab large objects, such as a basketball, with a single hand. The SR Fingers can help the person to accomplish those tasks.
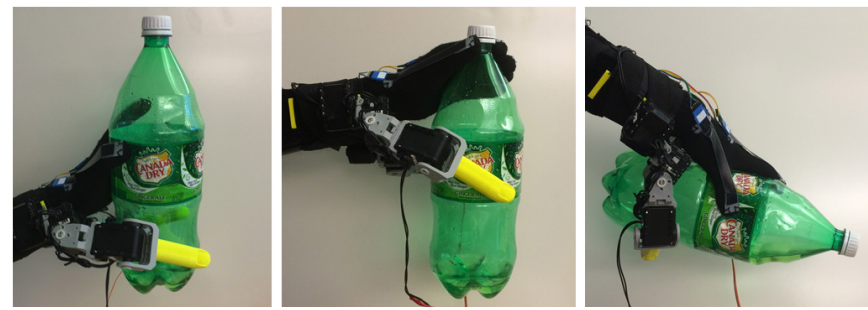

Figure 11. The SR Fingers can adapt to the user's grasping posture.

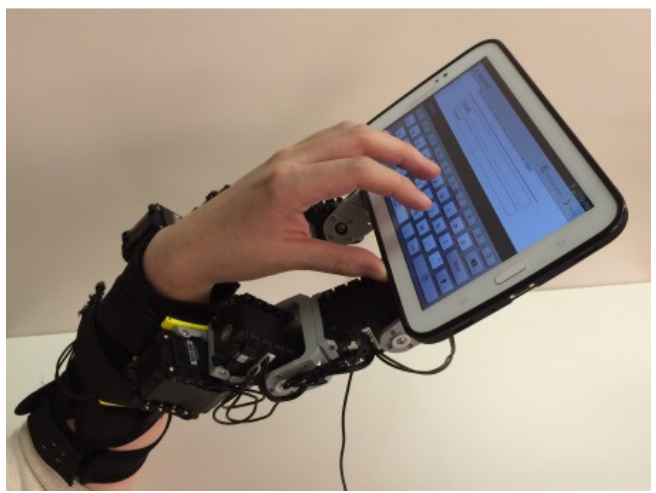

Figure 12. The 7-fingered hand can perform tasks that would usually require two hands, such as holding up a tablet computer and typing letters on it.

effective grasp, which can be optimized via traditional grasp theories, including grasp stability [49], form closure [50], force closure [51], and others [52].

The correlation control law may depend on the context, state, and other parameters of the task process, which means some higher-level description of the task, perhaps a backbone task model, is required to embed a collection of synergies in individual steps for more flexible task execution. Considering the natural and implicit nature of synergy-based control, we can view it as the lowest-level behavior in a hierarchical control system. Unless otherwise specifically requested or conditioned, the synergy-based control kicks in with a set of default parameter values. As more specific conditions and requirements are imposed, the synergy-based control must be 
modified, or switched to other synergies by a higher-level controller. Coloured Petri Net [53] may be used to integrate the bottom-up control method into a high-level, backbone controller consisting of discrete states.

The methodologies described in this paper can be extended to larger size SR Limbs in diverse tasks and contexts, such as manufacturing, construction, and elderly care. SR Limbs will be a promising and important branch of wearable robots that will infuse unique concepts of human-robot coordination. We hope this will provide a new direction for human-robot interaction and enable other assistive technologies to be developed in the future.

\section{REFERENCES}

[1] F. Parietti and H. Asada, "Dynamic analysis and state estimation for wearable robotic limbs subject to human-induced disturbances," Proc. of IEEE Int. Conference on Robotics and Automation, Karlsruhe, Germany, May 2013.

[2] F. Parietti and H. Asada, "Supernumerary robotic limbs for aircraft fuselage assembly: body stabilization and guidance by bracing," Proc. of IEEE Int. Conference on Robotics and Automation, Hong Kong, China, May 2014, in press.

[3] F. Parietti and H. Asada, "Bracing the human body with supernumerary robotic limbs for physical assistance and load reduction," Proc. of IEEE Int. Conference on Robotics and Automation, Hong Kong, China, May 2014, in press.

[4] B. Llorens - Bonilla, F. Parietti, and H. Asada, "Demonstration-based control of supernumerary robotic limbs," Proc. of IEEE/RSJ Int Conference on Intelligent Robots and Systems, pp. 3936-3942, 2012.

[5] B. Llorens - Bonilla, and H. Asada, "Control and coordination of Supernumerary Robotic Limbs based on human motion detection and task Petri Net," Pros. of ASME Dynamic Systems and Control Conference, 2013.

[6] N. Bernstein, The Coordination and Regulation of Movements, NY, Pergamon Press, 1967.

[7] M.L. Latash, "Toward a new theory of motor synergies," Motor Control, vol. 11, pp. 276-308, 2007.

[8] A. d'Avella, P. Saltiel, and E. Bizzi, "Combinations of muscle synergies in the construction of a natural motor behavior," Nature Neuroscience, vol. 6, pp. 300 - 308, 2003.

[9] A. d'Avella, A. Portone, and F. Lacquaniti, "Superposition and modulation of muscle synergies for reaching in response to a change in target location," J. Neurophysiol., vol. 106, pp. 2796-2812, 2011.

[10] Y.P. Ivanenko, R.E. Poppele, and F. Lacquaniti, "Five basic muscle activation patterns account for muscle activity during human locomotion," J. Physiol., vol. 556, pp. 267-282, 2004.

[11] A. d'Avella, P. Saltiel, and E. Bizzi, "Combinations of muscle synergies in the construction of a natural motor behavior," Nature Neuroscience, vol. 6, pp. 300 - 308, 2003.

[12] M. Tresch, P. Saltiel, and E. Bizzi, "The construction of movement by the spinal cord," Nature Neuroscience, vol. 2, pp. 162 - 167, 1999.

[13] T. Drew, J. Kalaska, and N. Krouchev, "Muscle synergies during locomotion in the cat: a model for motor cortex control," J Physiol, vol. 586, no. 5, pp. 1239-1245, March 2008.

[14] J. Winters, and P.E. Crago (Eds.), Biomechanics and Neural Control of Posture and Movement, Springer-Verlag, 2000.

[15] M.L. Latash, A.S. Alexander, and V.M. Zatsiorsky, "The basis of a simple synergy: recnstruction of joint equilibruium trajectories during unrestrained arm movement," Human Movement Science, vol. 18, pp.330, 1999.

[16] D. Prattichizzo, M. Malvezzi, M. Gabiccini, and A. Bicchi, "On motion and force controllability of precision grasps with hands actuated by soft synergies," Robotics, IEEE Transactions on, vol.29, no.6, pp.1440-1456, Dec. 2013.
[17] C. Grégoire, C. Papaxanthis, and M. Schieppati, "Coordinated modulation of locomotor muscle synergies constructs straight-ahead and curvilinear walking in humans," Experimental brain research, vol. 170, no.3, pp. 320-335, 2006.

[18] A. d'Avella, and E. Bizzi, "Shared and specific muscle synergies in natural motor behaviors," Proc. of the National Academy of Sciences of the United States of America, vol. 102, no. 8, pp. 3076-3081, 2005.

[19] H. Suenaga, Y. Hashizume, and J. Nishii, "An analysis of leg joint synergy during backward walking," Proc. of 35th Annual International Conference of the IEEE Engineering in Medicine and Biology Society, Osaka, Japan, 2013.

[20] M. Santello, M. Flanders, and J.F. Soechting, "Postural Hand Synergies for Tool Use," Journal of Neuroscience, vol. 18, pp. 10105-10115, Dec.1998.

[21] N. Kang, M. Shinohara, V.M. Zatsiorsky, and M.L. Latash, "Learning multi-finger synergies: an uncontrolled manifold analysis," Exp Brain Res, vol. 157, pp. 336-350, 2004.

[22] M.L. Latash, J.K. Shim, and V.M. Zatsiorsky, "Is there a timing synergy during multi-finger production of quick force pulses?" Exp Brain Res, vol. 159, pp. 65-71, 2004.

[23] Z.M. Li, M.L. Latash, and V.M. Zatsiorsky, "Force sharing among fingers as a model of the redundancy problem," Exp Brain Res, vol. 119, pp.276-286, 1998.

[24] M. Santello, and J.F. Soechting, "Force synergies for multifingered grasping," Exp Brain Res, vol. 133, pp. 457-467, 2000.

[25] J.K. Shim, M.L. Latash, and V.M. Zatsiorsky, "Prehension synergies: trial-to-trial variability and principle of superposition during static prehension in three dimensions," J Neurophys, vol. 93, pp. 3649-3658, 2003

[26] M.B. Popovie, D.B. Popovie, and R. Tomovie, "Control of arm movement: Reaching synergies for neuroprosthesis with life-Like control," J. Automatic Control, vol. 12, pp. 9-15, 2002.

[27] C. Brown, and H. Asada, "Inter-finger coordination and posture synergies in robot hands via mechanical implementation of Principal Component Analysis," Proc. of 2007 IEEE/RSJ Int. Conference on Intelligent Robots and Systems, October 2007.

[28] J. Rosmarin, and H. Asada, "Synergistic design of a humanoid hand with hybrid DC motor - SMA array actuators," Proc. of 2008 IEEE Int. Conference on Robotics and Automation, pp. 773-779, May 2008.

[29] W. Chen, and C. Xiong, "A principle of mechanical implementing the kinematic synergy for designing anthropomorphic hand," Proc. of the Intelligent Robotics and Applications. 6th International Conference, Busan, South Korea, September 2013.

[30] K. Xu, J. Zhao, Y. Du, X. Sheng, and X. Zhu, "Design and postural synergy synthesis of a prosthetic hand for a manipulation task," Proc. of the IEEE/ASME International Conference on Advanced Intelligent Mechatronics, Wollongong, Australia, July 2013.

[31] M. Ciocarlie, C. Goldfeder, and P. Allen, "Dimensionality reduction for hand-independent dexterous robotic grasping," Proc. of IEEE/RSJ Int. Conf. Intell. Robot. Syst., pp. 3270-3275, 2007.

[32] M. Ciocarlie, and P. Allen, "Hand posture subspaces for dexterous robotic grasping," Int. J. Robot. Res., vol. 28, pp. 851-867, 2009.

[33] T. Wimboeck, B. Jan, and G. Hirzinger, "Synergy-level impedance control for a multifingered hand," Proc. of IEEE Int. Conf. Robot. Autom., pp. 973-979, 2011

[34] A. Miller, and P. Allen, "Graspit!: A Versatile Simulator for Robotic Grasping," IEEE Robotics and Automation Magazine, vol. 11, no.4, pp. 110-122, December 2004.

[35] G. Gioio, G. Salvietti, M. Malvezi, and D. Prattichizzo, "Mapping synergies from human to robotic hands with dissimilar kinematics: an approach in the object domain," IEEE Transactions on Robotics, vol. 29, no. 4, pp. 825-37, August 2013.

[36] T. Geng, M. Lee, and M. Hulse, "Transferring human grasping synergies to a robot," Mechatronics, vol. 21, no. 1, pp. 272-284, February 2011.

[37] R. Vinjamuri, S. Mingui, C. Cheng-Chun, L. Heung-No, R.J. Sclabassi, and M. Zhi-Hong, "Dimensionality reduction in control and coordination of the human hand," IEEE Trans. Biomed. Eng., vol. 57, no. 2, pp. 284295, 2010. 
[38] N. Karnati, B.A. Kent, and E.D. Engeberg, "Bioinspired sinusoidal finger joint synergies for a dexterous robotic hand to screw and unscrew objects with different diameters," Mechatronics, IEEE/ASME Transactions on , vol.18, no.2, pp.612,623, April 2013.

[39] S. Schaal, "Learning rhythmic movements by demonstration using nonlinear oscillators," IEEE/RSJ International Conference on Intelligent Robots and System, vol. 1, pp. 958-963, 2002.

[40] R. Ronsse, B. Koopman, N. Vitiello, T. Lenzi, S.M.M. De Rossi, J. van den Kieboom, E. van Asseldonk, M.C. Carrozza, H. van der Kooij, and A.J. Ijspeert, "Oscillator-based walking assistance: A model-free approach," Rehabilitation Robotics (ICORR), 2011 IEEE International Conference on , pp.1-6, June 2011.

[41] J. McNeil, "Americans with disabilities: household economic studies," United States Census, pp. 70-73, Feburary 2011.

[42] C.L. MacKenzie, and T. Iberall, The Grasping Hand, North-Holland, Amsterdam, 1994.

[43] P.K. Levangie, and C.C. Norkin, Joint Structure and Function: A comprehensive Analysis, 3rd ed., pp. 265-285, Philadelphia, PA: F.A Davis Company, 1992.

[44] A. Kargov, C. Pylatiuk, J. Martin, S. Schulz, and L. Doderlein, "A comparision of the grip force distribution in natural hands and in prosthetic hands, " Disabil Rehabil, vol. 26, no. 12, pp. 705-711, 2004.

[45] V. Bundhoo, and E. J. Park, "Design of an artificial muscle actuated finger towards biomimetic prosthetic hands," Proc. of 12th Inte. Conf. on Advanced Robotics, pp.368-375, 2005.
[46] S. Wold, A. Ruhe, H. Wold, and W.J. Dunn, "The collinearity problem in linear regression: the partial least squares (PLS) approach to generalized inverses," SIAM Journal on Scientific and Statistical Computing, vol. 5, no. 3, pp. 735-743, 1984.

[47] H. Hanafusa, and H. Asada, "Stable pretension by a robotic hand with elastic fingers," Proc. of 7th Int. Symp. on Industrial Robots, pp. 361367, October 1977.

[48] K. Salisbury, and J.J. Craig, "Articulated hands: force control and kinematic issues," Int. J. Robotics Research, vol. 1, no. 1, pp. 4-17, 1982

[49] H. Hanafusa, and H. Asada, "Stable Pretension by a Robotic Hand with Elastic Fingers," Proc. of 7th Int. Symp. on Industrial Robots, pp. 361367, October 1977.

[50] K. Lakshminarayana, "Mechanics of Form Closure," ASME Technical paper 78-DET-32, 1978.

[51] E. Rimon, and J. Burdick, "On Force and Form Closure for Multiple Finger Grasps", Proc. of 1996 IEEE International Conference on Robotics and Automation, 1996.

[52] A. Sudsang, J. Ponce, and N. Srinivasa, "Grasping and In-Hand Manipulation: Experiments with a Reconfigurable Gripper", Advanced Robotics, 1998.

[53] K. Jensen, and L.M. Kristensen, Coloured Petri Nets: Modelling and Validation of Concurrent Systems, Springer, 2009. 